\title{
THERMAL DESIGN OF ENCLOSURE FOR ELECTRIC BASEBOARD HEATERS
}

\author{
Ruoyao $\mathrm{Li}^{1}, \mathrm{Ri} \mathrm{Li}^{2 *}$ \\ ${ }^{1}$ School of Engineering, The University of British Columbia, Kelowna, BC, Canada \\ *sunny.li@ubc.ca
}

\begin{abstract}
Electric baseboard heaters have been widely used to provide convenient and economical heating in residential rooms. For old heaters that were installed many years ago, a secondary enclosure can be installed to cover the old heater for aesthetic and preventing scalding by the hot elements. The enclosure increases the thermal resistance for heating, which results in high temperature on the top surface of the enclosure. There is a significant lack of the information and work on the thermal design of enclosure for electric baseboard heaters. Developing an effective enclosure design that is able to keep the surface temperature within safe-to-touch range is the objective of this research. In this project, computational fluid dynamics simulation using COMSOL Multiphysics is the major methodology. Three approaches are taken to develop the new enclosure design. The first approach is to change material's thermal properties: thermal conductivity, surface emissivity. It was found that the surface temperature decreased with reducing the thermal conductivity of the material. Moreover, the surface temperature can be effectively reduced by using the high surface emissivity coating material on the outer surface and the low surface emissivity coating material on the inner surface. The second approach is to modify mechanical design to facilitate air flow. It was discovered that increasing the openings for air inlet and outlet can effectively reduce the surface temperature. The third approach is to use thermoelectric fan to provide passively-powered forced convection heat transfer. It was found that the thermoelectric fan can be used to not only enhance the airflow through the heater but also to reduce the top surface temperature of the enclosure. The three approaches will be combined to develop effective and safe enclosures for electric baseboard heaters.
\end{abstract}

